\title{
Research Paper: \\ The Effect of Ganoderma Supplementation and Selected Exercise Training on Glycemic Control in Boys With Type 1 Diabetes
}

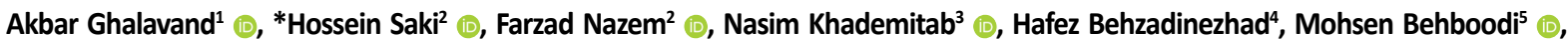 \\ Fatemeh Zeihami ${ }^{4}$
}

1. Pediatric Gastroenterology and Hepatology Research Center, Zabol University of Medical Sciences, Zabol, Iran. 2. Department of Physical Education and Sports Science, Faculty of Sports Sciences, Bu-Ali Sina University, Hamedan, Iran.

3. Department of Physical Education and Sports Science, Faculty of Sports Sciences, Tehran University, Tehran, Iran.

4. Department of Physical Education and Sports Science, Takestan Branch, Islamic Azad University, Takestan, Iran.

5. Department of Physical Education and Sports Science, Faculty of Sports Sciences, Hakim Sabzevari University, Sabzevar, Iran.

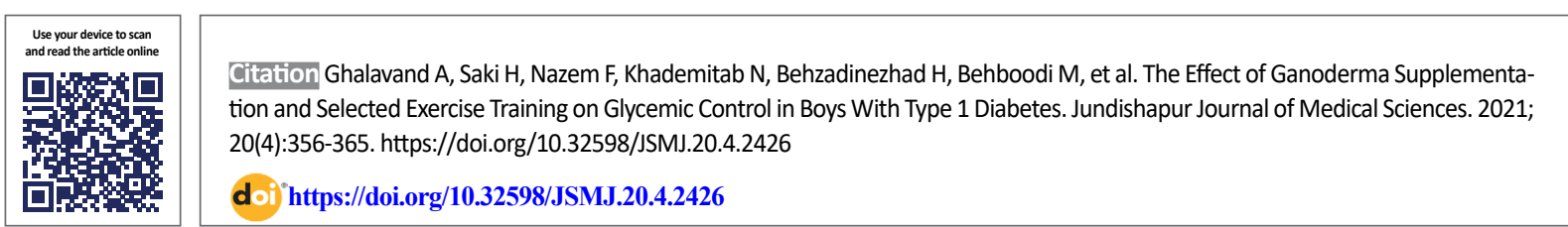

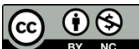

Received: 27 Mar 2021

Accepted: 13 Aug 2021

Available Online: 01 Oct 2021

Keywords:

Type 1 diabetes,

Exercise training,

Ganoderma, Glyce-

mic control

\section{ABSTRACT}

Background and Objectives Type 1 diabetes is one of the most common chronic diseases in children. The aim of this study was to determine the effect of eight weeks of regular exercise training and Ganoderma supplementation on glycemic control in children with type 1 diabetes.

Subjects and Methods In this quasi-experimental study, 40 boys with type 1 diabetes were selected and divided into 4 groups: 1) exercise, 2) supplement, 3) exercise \& supplement 4) control. Exercises included 8 weeks, 3 sessions per week and each training session included selected exercises training. Ganoderma lucidum consumption for daily consumption of 20 grams of a brewed drink was consumed. Fasting blood sampling was performed 48 hours before and 72 hours after the intervention.

Results A significant decrease was observed in 2 Hour Post Prandial Glucose in the exercise \& supplement group compared to the control group ( $\mathrm{P}=0.018)$. Also Significant decrease was observed in $\mathrm{HbA} 1 \mathrm{c}$ level in exercise $(P=0.031)$ and exercise \& supplement $(P=0.043)$ compared to the control group.

Conclusion According to the results, it can be said that combined exercise training can play an important role in the management of blood sugar in type 1 diabetes and the use of Ganoderma can increase the effectiveness of exercise in blood glycemic control along with exercise training.

\section{* Corresponding Author:}

Hossein Saki

Address: Department of Physical Education and Sports Science, Faculty of Sports Sciences, Bu-Ali Sina University, Hamedan, Iran.

Tel: +98 (916) 7016834

E-Mail: hossein.saki1990@yahoo.com 


\section{اثر مكمل تَانودرما و تمرينات ورزشى منتخب بر كنترل تَليسمى در يسران مبثلا به ديابت نوع}

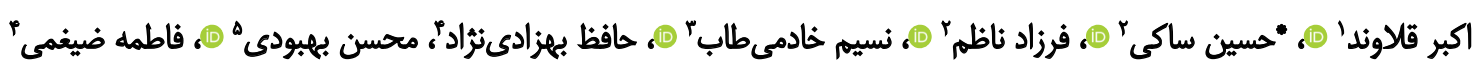

ا. ا. مركز تحقيقات كوارش و كبد كودكان، دانشكاه علوم يزشكى زابل، زابل، ايران.

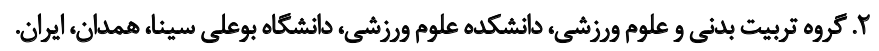

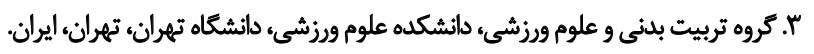

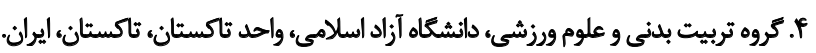

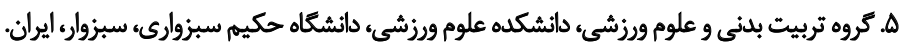

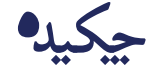

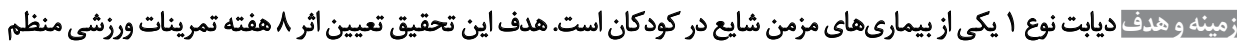

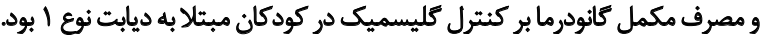

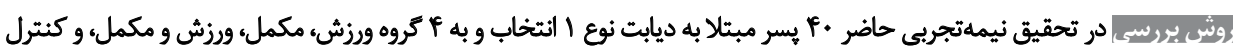

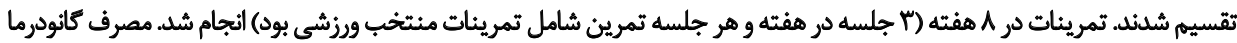

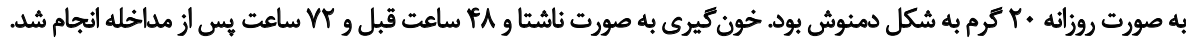

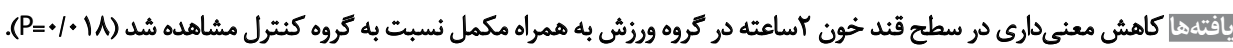

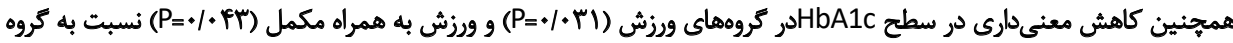
كنترل مشاهد شد.

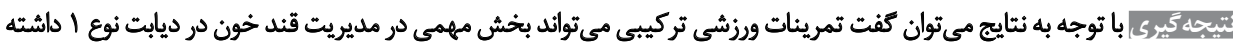

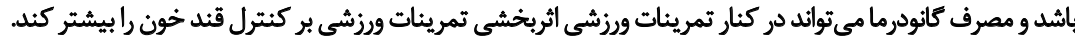

Fريخ

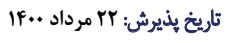

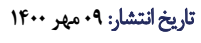

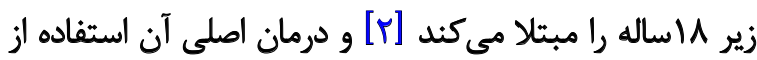

daleo

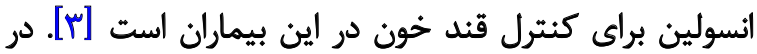

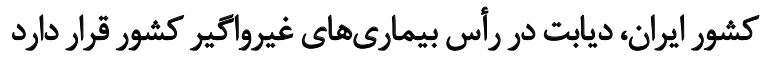

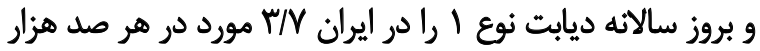

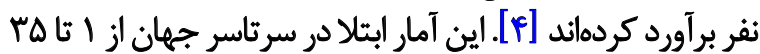

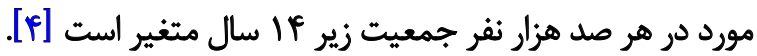

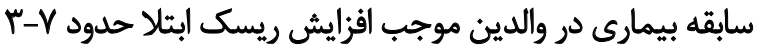

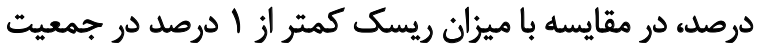

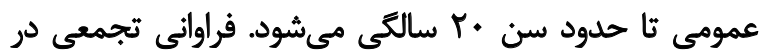

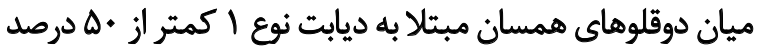

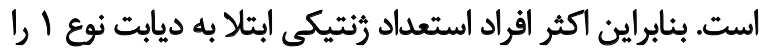

ديابت شيرين نوع ا يكى از بيمارىهاى متابوليك است كه در

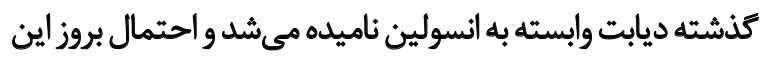

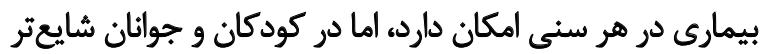

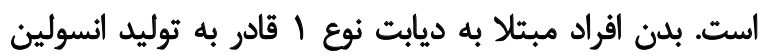

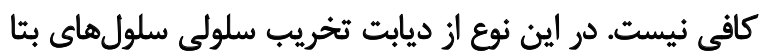

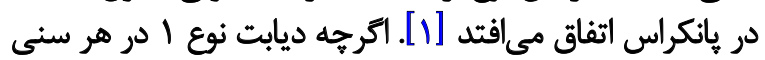

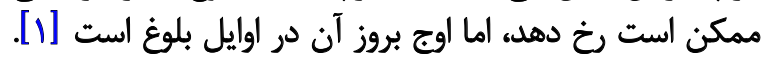

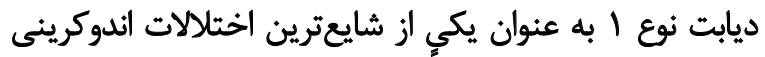

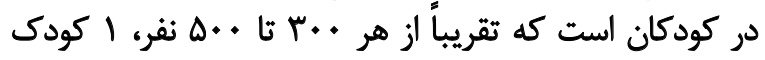

a..........

$$
\begin{aligned}
& \text { * تويسينده مسيئول: } \\
& \text { حسين ساكى } \\
& \text { نشاني: همداني، دانشكَاه بوعلي سينا، دائشكده علوم ورزُشي، كروه تربيت بلني و علوم ورزشي. } \\
& \text { تلفئ: } \\
& \text { راياثامه: hossein.saki1990@yahoo.com }
\end{aligned}
$$


يكى ديكر از روشهاى كنترل كليسميك در بيماران ديابتى إنى

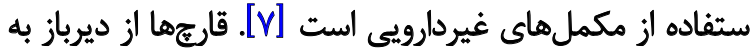

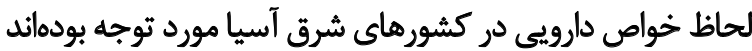

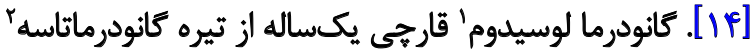

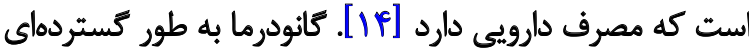

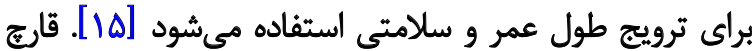

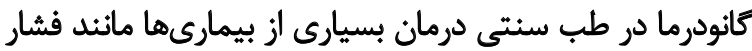

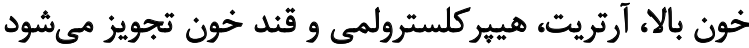

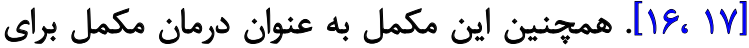

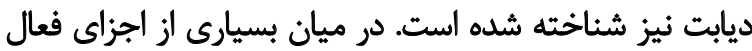

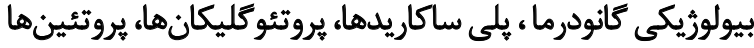

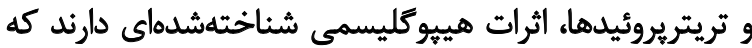

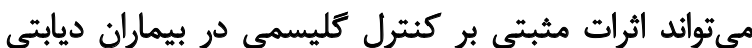

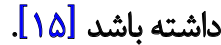

استفاده از تمرينات ورزشى و مكمل كانودرما با توجه به اثرات

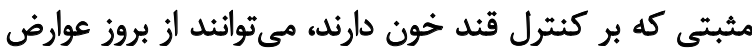

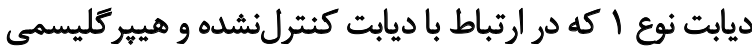

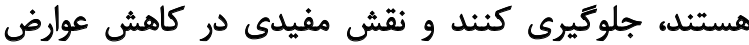

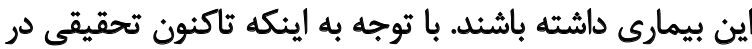

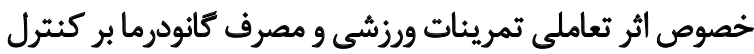

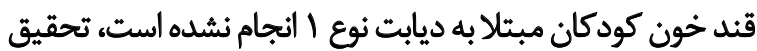

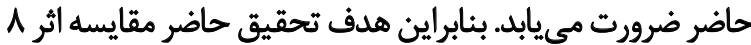

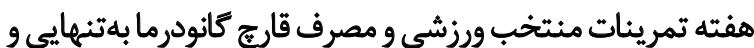

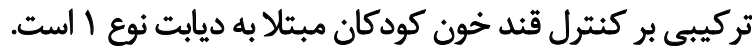

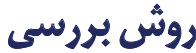

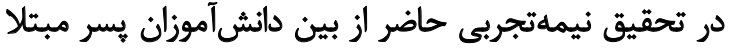

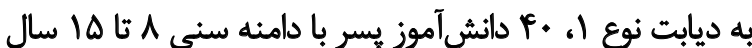

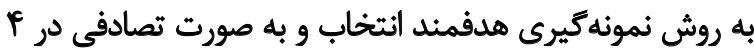

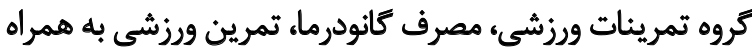
مصرف كانودرما وكنترل تقسيم شدند.

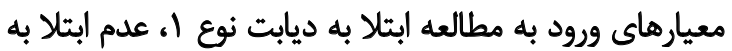
مشكلات قلبى تنفسى حاد، عدم ابتلا به بيمارى هائ مزئه

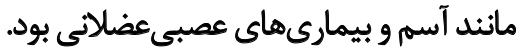

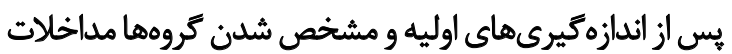

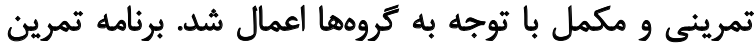

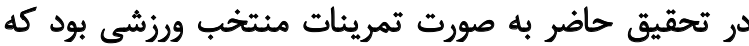

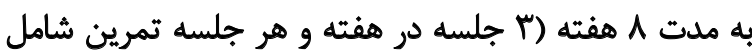

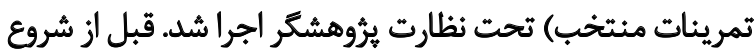

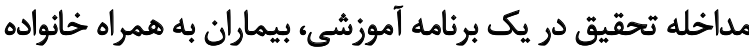

1. Ganoderma lucidum Karst

2. Ganodermataceae

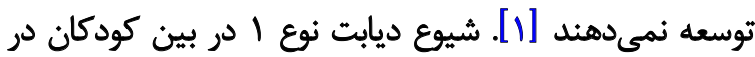

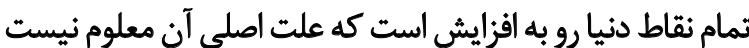

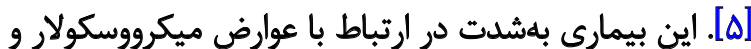

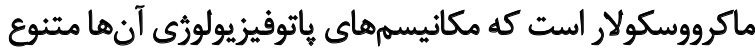

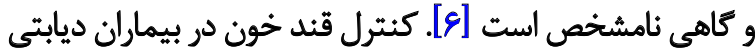

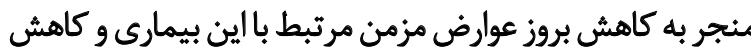

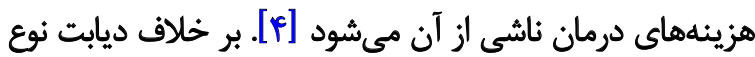

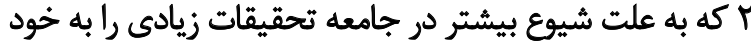

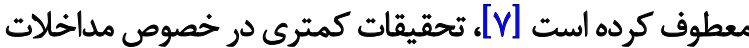

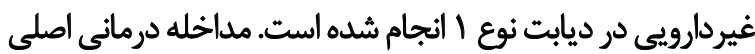

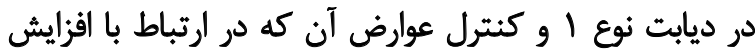

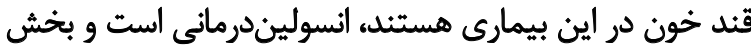

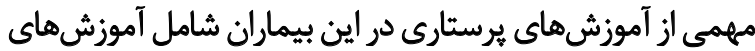

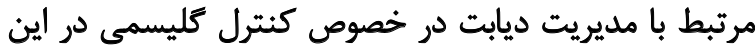

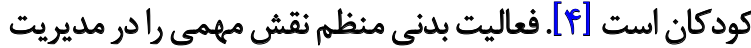

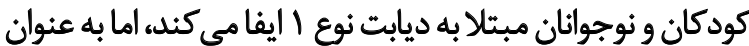

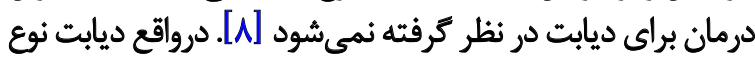

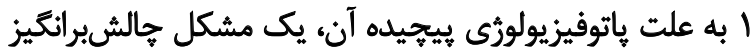

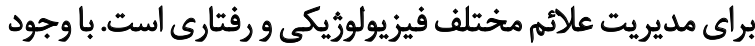

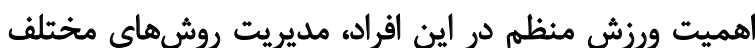

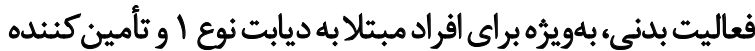

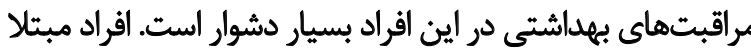

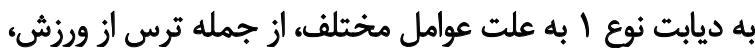

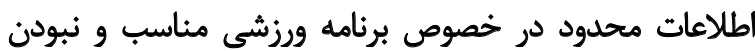

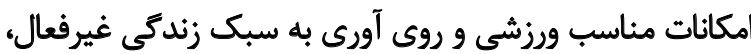

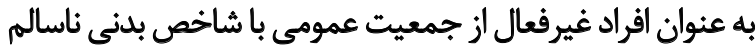

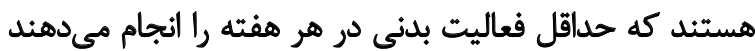

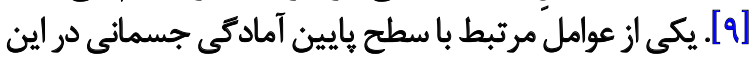

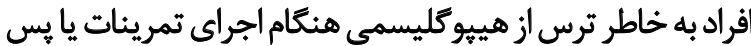

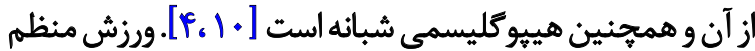

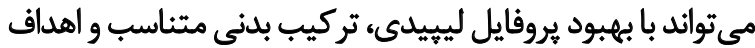

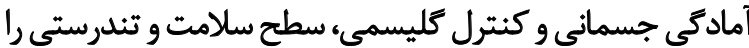

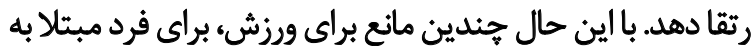

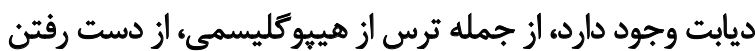

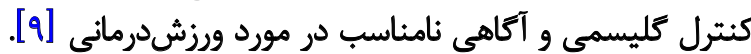

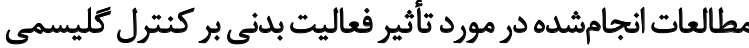

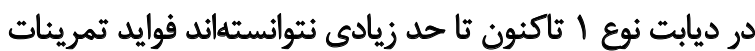

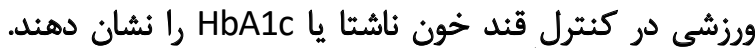

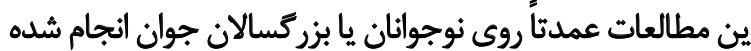

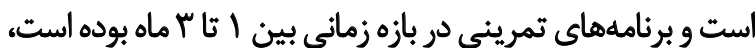

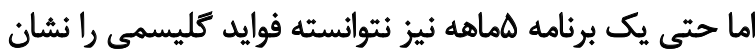

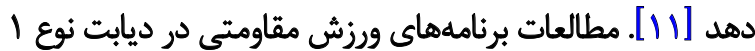

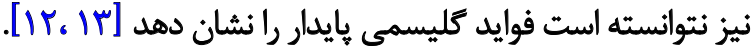

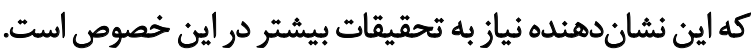




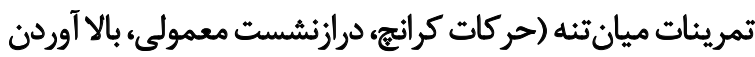

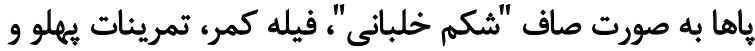

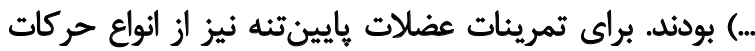

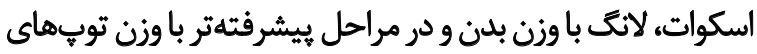

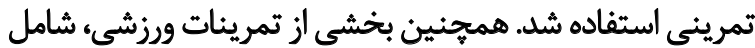

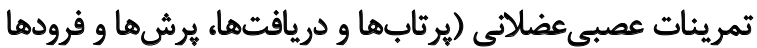

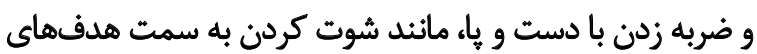

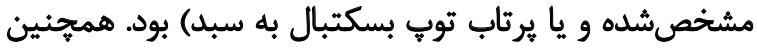

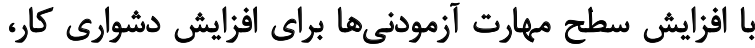

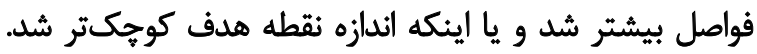

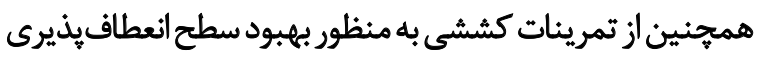

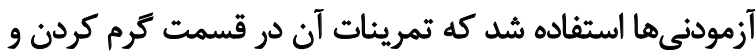
در إيان قسمت سرد كردن قرار داده شده بودات درد

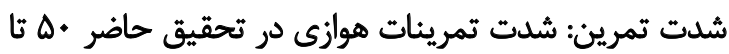

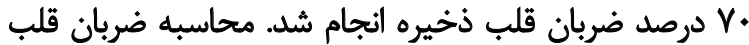

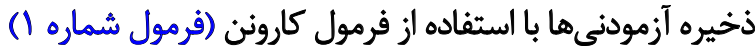

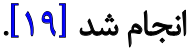

$$
\begin{aligned}
& \text { سن - • - Y = ضربان قلب بيشينه } \\
& \text { ضربان قلب استراحت + [درصد شدت × (ضربان قلب استراحت } \\
& \text { - ضربان قلب بيشينه)] = ضربان قلب هدف الدرمد }
\end{aligned}
$$

ج) سرد كردن: مدت اين مرحله ها الى • ( دقيقه بود. آزمودنى

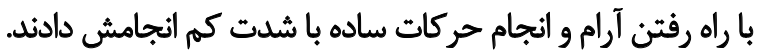

در كروهاي مصرف مكمل كانودرما و تمرين به همراه مكمل

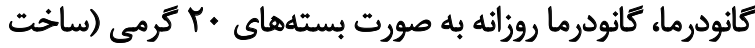

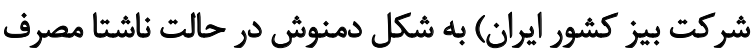

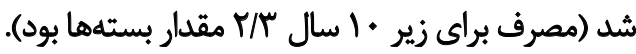

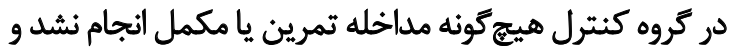

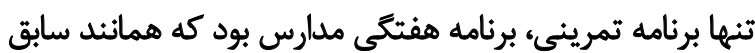

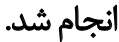

جهت بررسى متغيرهاى وابسته خون ميرى در دو مرحله

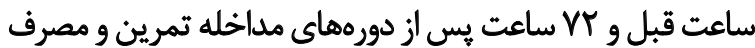

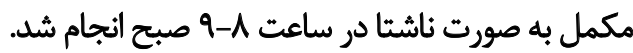

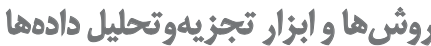

براى تجزيهوتحليل دادهها از آمار توصيفى كه شامل ميانكين

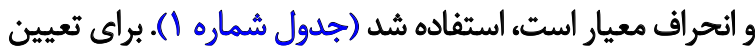

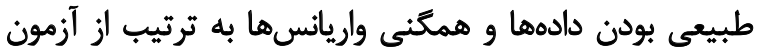

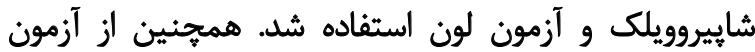

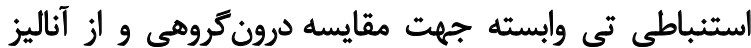

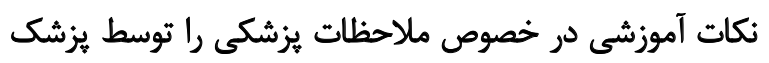

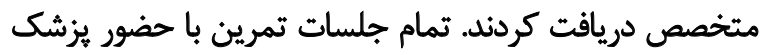

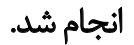

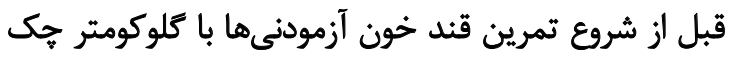

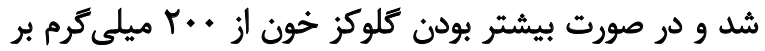

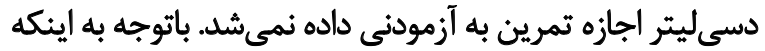

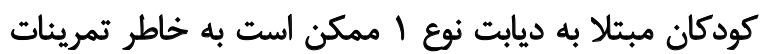

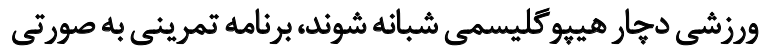

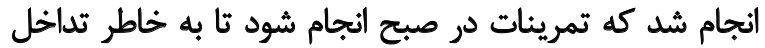

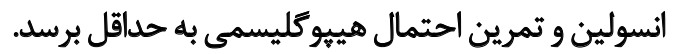

جلسات تمرينى در تحقيق حاضر شامل سه بخش زير بود: الف) ترم كردن: طول مدت ترم كردن • الى الى ها دقيقه

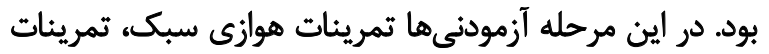

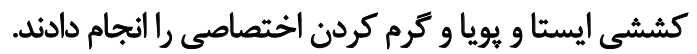

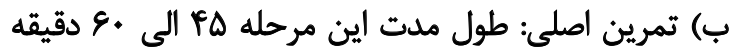

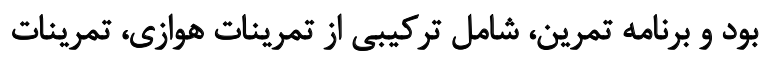

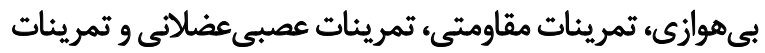

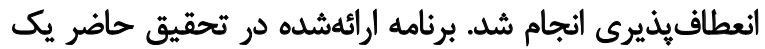

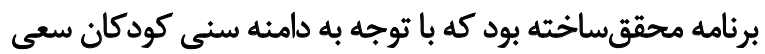

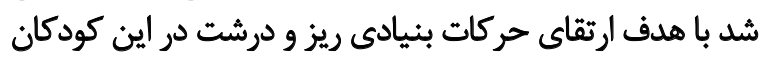

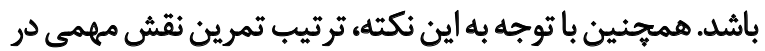

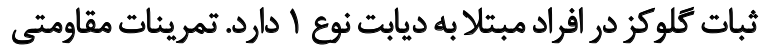

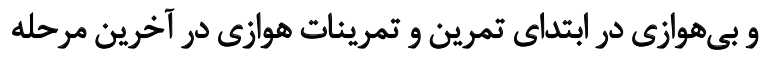

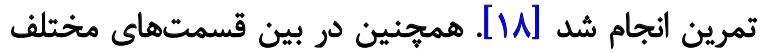

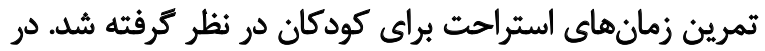

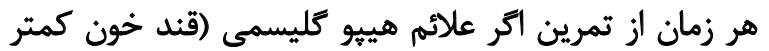

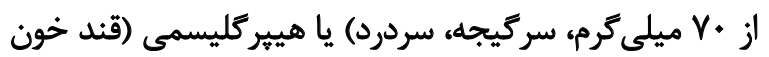

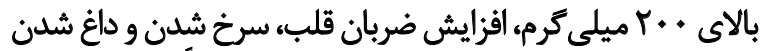

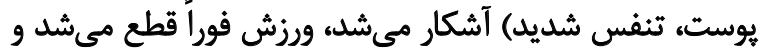

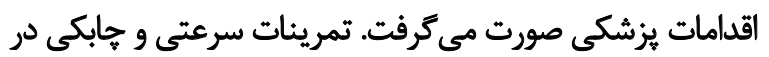

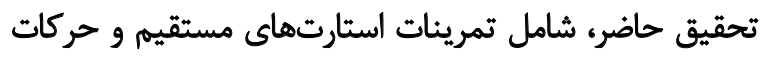
جابكى شامل جابهجايى، مانند دويدن و وعبور از بين موانع بات تغينير

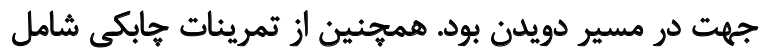

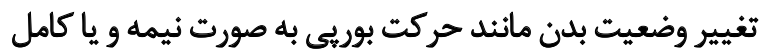

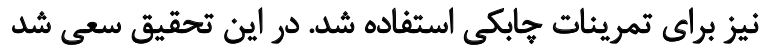

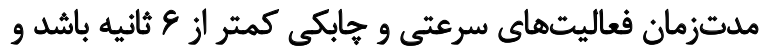

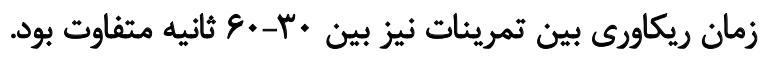

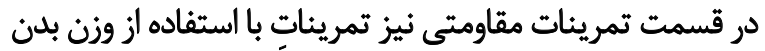

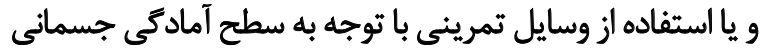

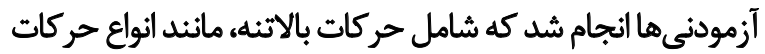

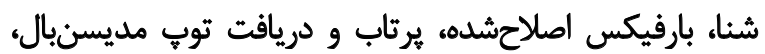

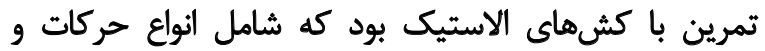




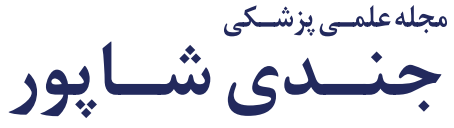

جدول ا. مشخصات جمعيثشئاختى و تنسنجى گروهاي تحقيق

\begin{tabular}{|c|c|c|c|c|}
\hline \multicolumn{4}{|c|}{ ميانين } & \multirow{2}{*}{ كتووه } \\
\hline درصد جربى بدن & قد & وزن & سن & \\
\hline WNTrET/RA & $\mid T T / / V \pm I T / T T$ & rNFA土VAF & $\mid 1 / r r \pm T / A 1$ & ورزش + كانودرما \\
\hline$|F / r \Psi \pm \pi / m|$ & $I \pi / F V \pm V \cdot V$ & $r g / M+ \pm r / M$ & $1 . / A \Psi \pm r / q \Psi$ & كانودرما \\
\hline $1 \Delta / N \pm r / \Delta r$ & $|r q / 8 . \pm 1 F| q q$ & $r / / T+\varepsilon / 9 q$ & $1 W / \Delta \cdot \pm r / V$. & ورزش \\
\hline $1 Q M T \pm r / M r$ & $|f| / . . \pm t \psi \cdot \cdot \Delta$ & HNIVET/AY & $\mid W / \Delta T \pm T / E T$ & كتترل \\
\hline
\end{tabular}

شد، اما ثناوت معنى دارى در سطوح قند خون ناشتًا (P=/ / FY)

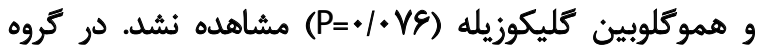

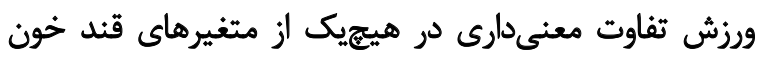

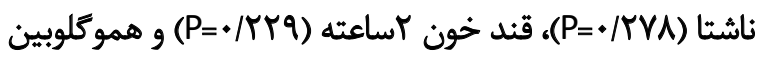

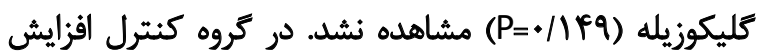

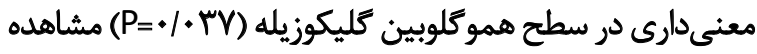

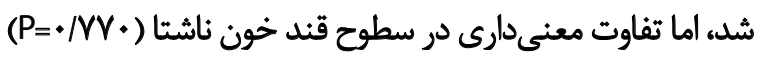

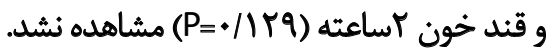

در بررسي تغييرات بينگروهي، نتايج آزمون تحليل واريانس

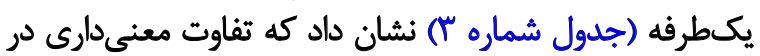

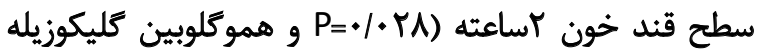
بين كروهاى تحقيق وجود داشت، اما تثاوت
واريانس يكسرفه و آزمون تعقيبى ثوكى براى مقايسه تغييرات

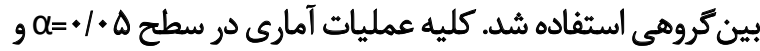

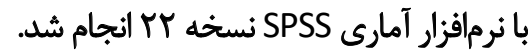

batist

در بررسى ثغييرات درون كروهى، نتايج آزمون تي وابسته

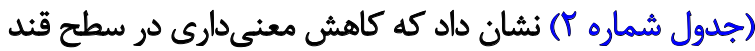

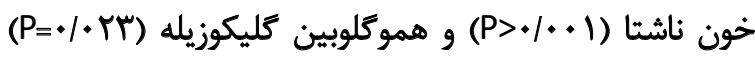
در كروه تركيبى تمرين ورزشى به همراه مصرف كافئ مانودرما

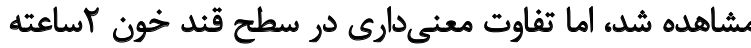

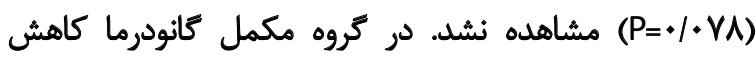

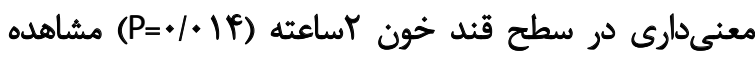

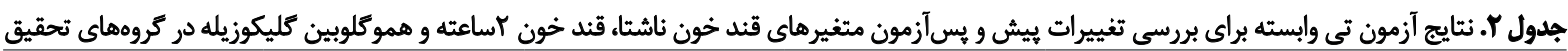

\begin{tabular}{|c|c|c|c|c|c|}
\hline \multirow{2}{*}{$\mathbf{P}$} & \multirow{2}{*}{$\mathbf{t}$} & \multicolumn{2}{|c|}{ ميانكين土|نحراف معيار } & \multirow{2}{*}{ تيروه } & \multirow{2}{*}{ مثغير } \\
\hline & & يسآزمون & يشي آزمون & & \\
\hline$<+/+\infty)$ & $11 / 797$ & $198 / 1 Y \pm V q / 8$ & 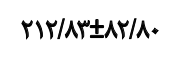 & ورزش + كانودرما & \multirow{4}{*}{ (ميلى قرم برون ناتشئي) } \\
\hline INFT & I/Me & IVA/rTINAT/OY & $|A F / \Delta \cdot \pm \lambda| / \cdot$ & كانودرما & \\
\hline.$/ r Y A$ & $1 / Y 1 Q$ & $r \cdot . / T \pm N W \cdot \Delta$ & $r \cdot q \cdot . \pm V \Delta / / \varepsilon$ & ورزش & \\
\hline.$/ W$ & $-\cdot \pi \cdot 1$ & $r \cdot \Delta / I Y \pm T T / M$ & $r+Y / \cdot \pm \pm Y V / T$. & كنترل & \\
\hline 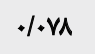 & $r / T+\varepsilon$ & TQT/RYIIINGT & TaT/rTIII/gr & ورزش + كانودرما & \multirow{4}{*}{ (ميلى كرم بر دسى لساعتي) } \\
\hline$\% 1 \%$ & $r / M$. & $m \Psi x / I V \pm|r| r \mid$ & $r E Q / \cdot . \pm M Y / \Delta T$ & كانودرما & \\
\hline.$/ \pi 9 q$ & $I / T \%$ & $r W / A r \pm I r+19 T$ & $\Psi A T / * \pm I M Y / \mathscr{N}$ & ورزش & \\
\hline.$/ 1 r q$ & $-1 / A 1 f$ & $r+\cdot / \lambda r \pm n r / A r$ & TWD.IVQ/FV & 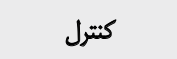 & \\
\hline 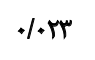 & T/TAI & $N F I \pm 1 / N$ & q/TEtr//r & ورزش + كانودرما & \multirow{4}{*}{ هموكلوبين كليكوزيله } \\
\hline H.ve & T/TMA & $V \cdot T \pm T / T V$ & $Q / T \pm T / \Delta$. & أنودرما & \\
\hline /NFq & $1 / V \cdot C$ & V..ET/TQ & จ१!ะพ/Tด & و ورزش & \\
\hline $.1 \cdot r r$ & -T/ATA & $q / T \Delta \pm V / T A$ & $N$ Vot+/Q1 & كنترل & \\
\hline
\end{tabular}


جدول "ا. نتايج آزمون تحليل واريانس يكطرفه براي مقايسه بين كروهى تغييرات قند خون ناشتا، قند خون باستته و هموكلوبين كليكوزيله

\begin{tabular}{|c|c|c|c|c|}
\hline \multirow{2}{*}{$\mathbf{P}$} & \multirow{2}{*}{$\mathbf{t}$} & ميانكين土|نحراف معيار & \multirow{2}{*}{ ت مروه } & \multirow{2}{*}{ 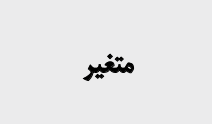 } \\
\hline & & ميزان تغييرات & & \\
\hline \multirow{4}{*}{. ME. } & \multirow{4}{*}{$1 / 4 r T$} & rIr/ArEAr/A. & ورزش + كاثودرما & \multirow{4}{*}{ 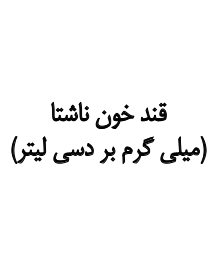 } \\
\hline & & $|M F / \Delta \cdot \pm \wedge| / \cdot \cdot$ & كانودرما & \\
\hline & & $r \cdot V \cdot+ \pm V \Delta / 1 \varepsilon$ & ورزش & \\
\hline & & $r \cdot r / \circ \pm \pm V / T$. & 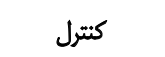 & \\
\hline \multirow{4}{*}{$.1 \cdot r A$} & \multirow{4}{*}{$r / 191$} & rqT/TTIIIV/R & ورزش + كاثودرما & \multirow{4}{*}{ 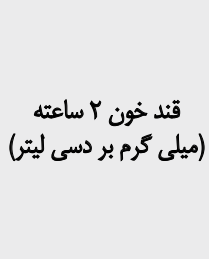 } \\
\hline & & $r E \Delta / . . \pm$ MY/AT & كانودرما & \\
\hline & & 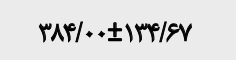 & 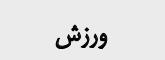 & \\
\hline & & rNA. IVQ/EV & كنترل & \\
\hline \multirow{4}{*}{.$\% 1 \mathrm{u}$} & \multirow{4}{*}{$\varphi / T$} & Я/צE & ورزش + كانودرها & \multirow{4}{*}{ هموكلويين كليكوزيله } \\
\hline & & $Q / T r \pm T / \Delta$. & كانودرما & \\
\hline & & จ११ $\pm T / r \Delta$ & ل ورزش & \\
\hline & & NVD士*/91 & كنترل & \\
\hline
\end{tabular}

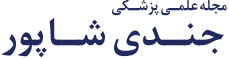

كه تمرينات ورزشى موجب كاهش سطح

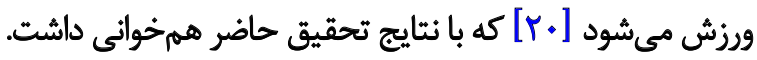

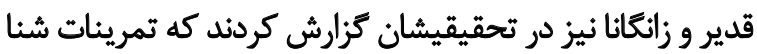

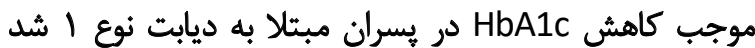

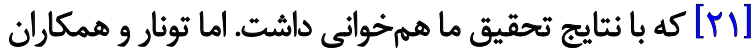

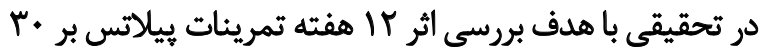

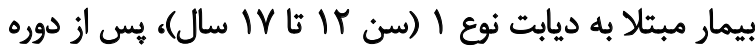

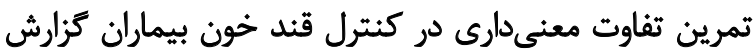

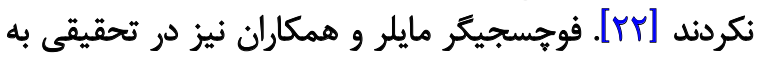

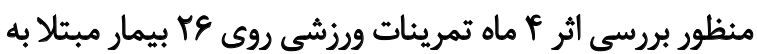

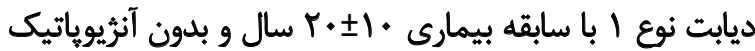

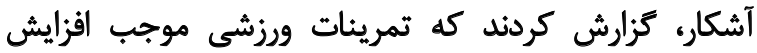
معنى دارى در سطح VO

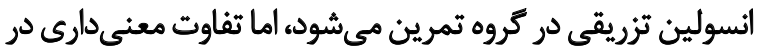

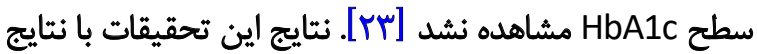

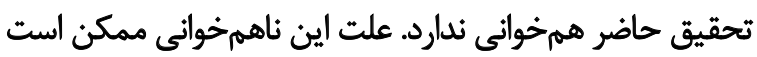

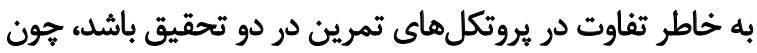

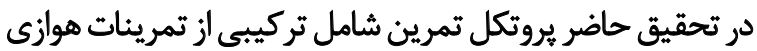
و تمرينات مقاومتي بود.

در خصوص رابطه بين فعاليت بدنى و كنترل كليسمى در

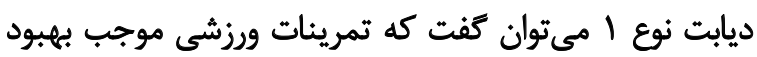

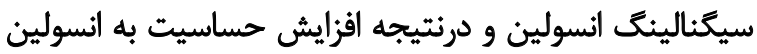

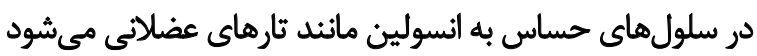

معنى دارى در قند خون ناشتا (•P=/T\&) مشاهده نشد.

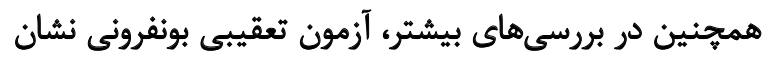

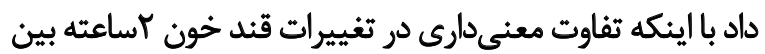

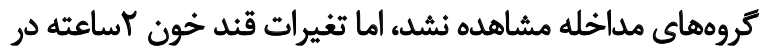

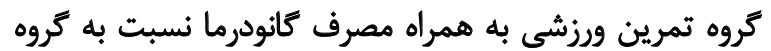

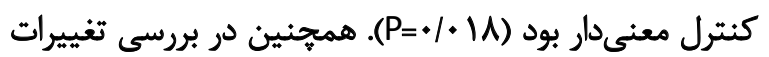

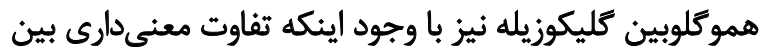

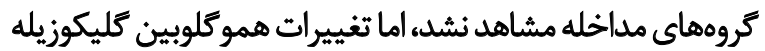

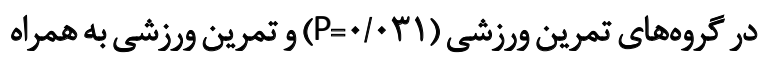

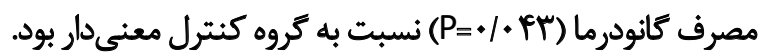

ب

در بررسى اثر تمرينات ورزشى بر كنترل كليسميك كودكان

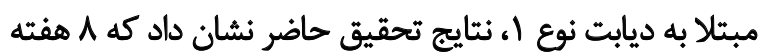

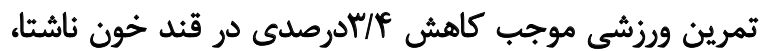

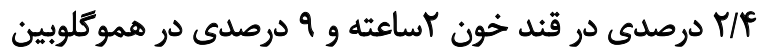
كليكوزيله شد و تغييرات هموكلوبين كليكوزيله نسبت بـ به

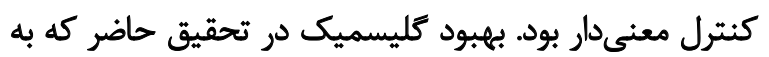

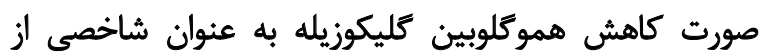

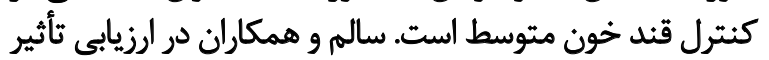

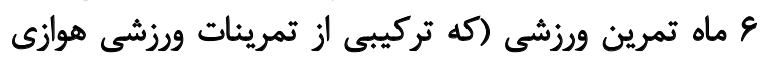

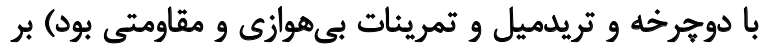

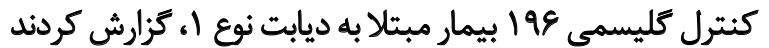


مورد بروهش در اين تحقيقات كه تحقيقاتى حيوانى بودند با بان باني

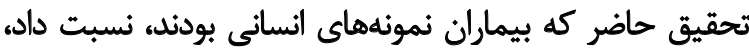

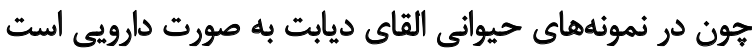

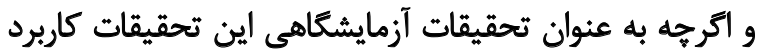

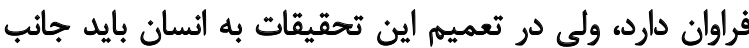

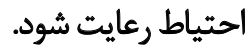

در تروه تركيبى كه در كنار 1 هفته تمرين ورزشى، مكمل كانودرما مصرف كرده بودند نيز كاهش ترد

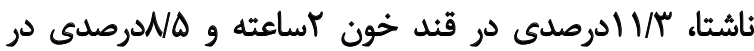

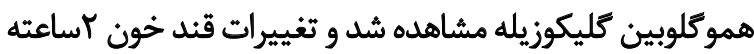

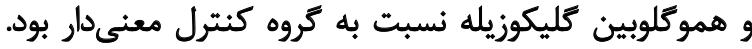

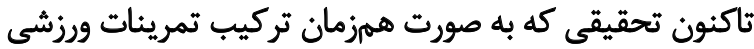

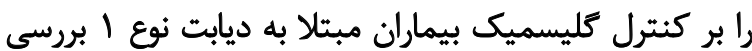

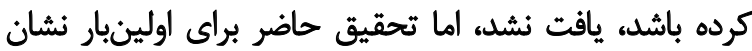

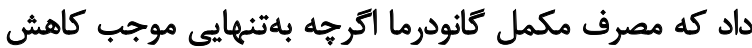

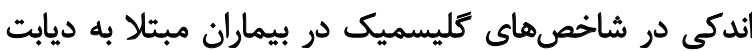

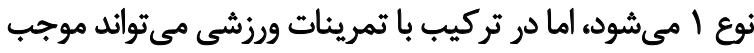

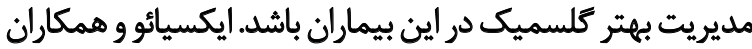

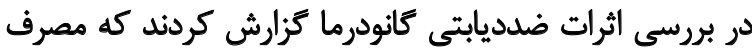

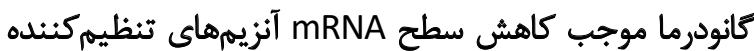

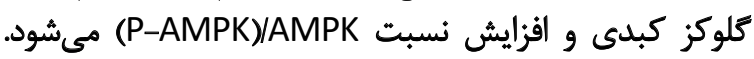

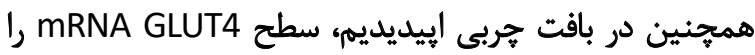

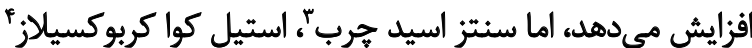

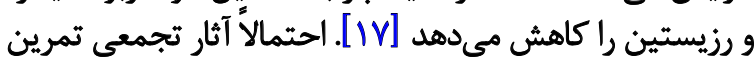

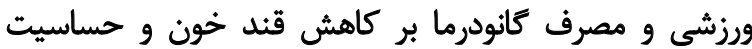

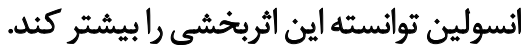

\section{نتيجليَّىى}

در كل يافتههاى تحقيق حاضر نشان داد كه مصرف 1 هفته

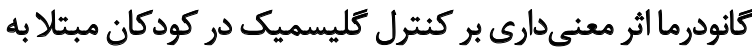

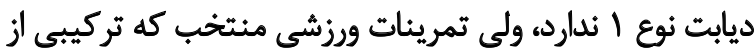

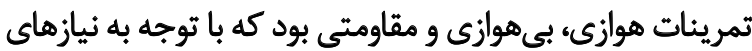

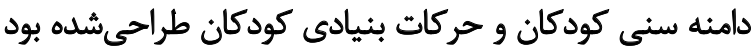

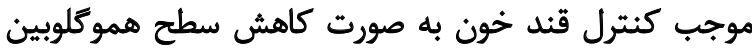

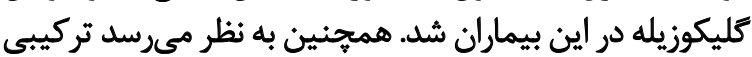

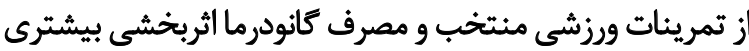
نسبت به هر روش بهتنهايي دارد.

3. Fatty acid synthase

4. Acetyl-CoA Carboxylase (ACC1)

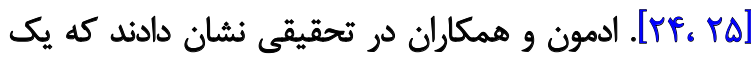

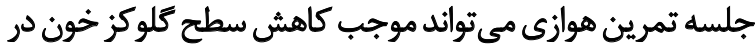

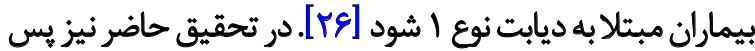

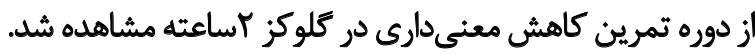

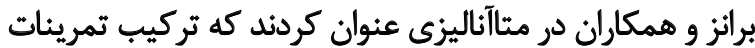

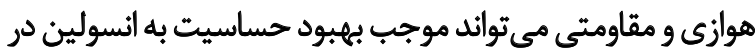

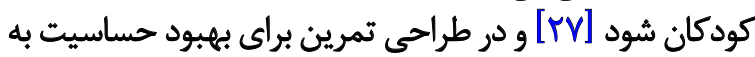

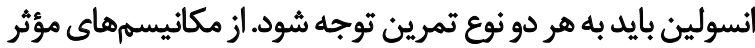

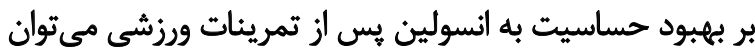

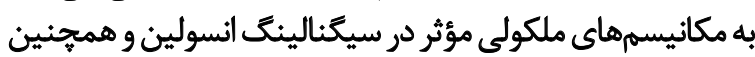

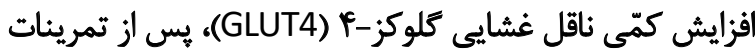

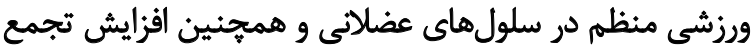

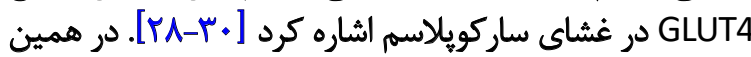

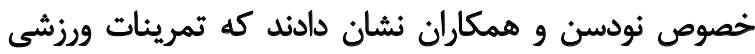

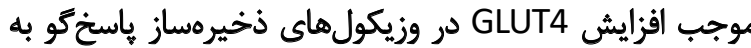

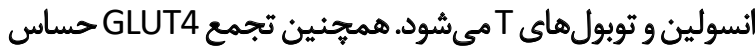

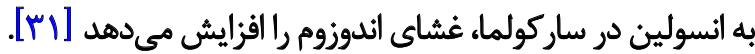

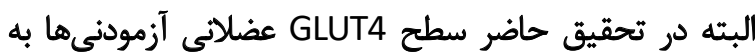

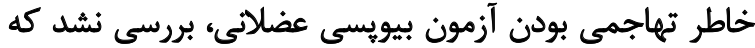

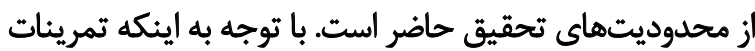

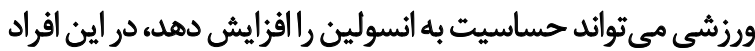

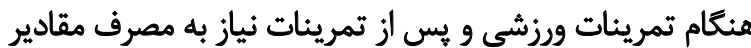

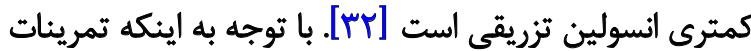

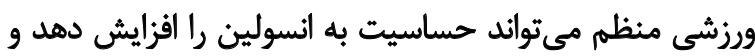

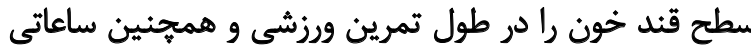

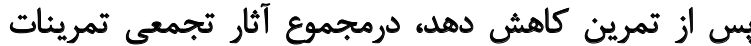

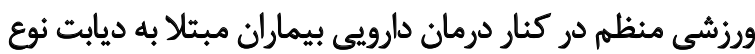

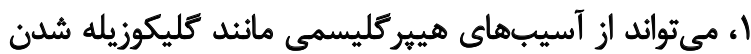

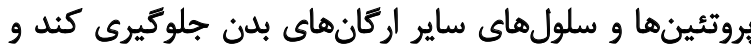

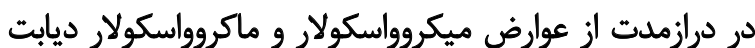

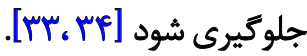

در گروه مصرف مصرف مكمل كانودرما كاهش

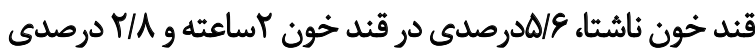

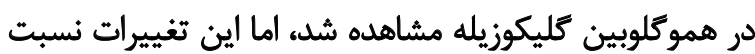

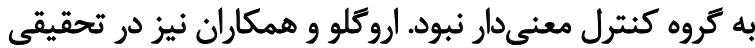

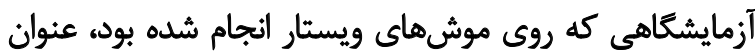

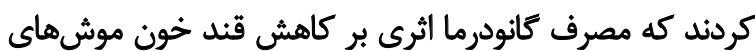

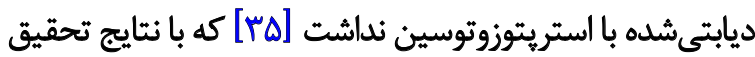

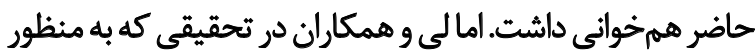

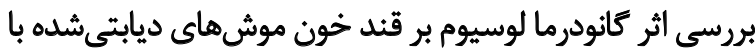

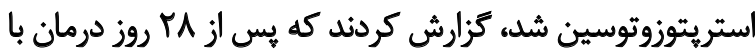

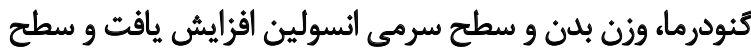

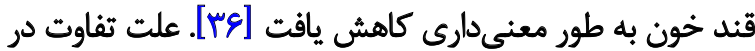

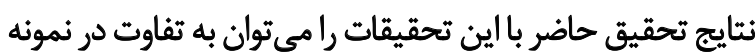


تمامى مراحل يرؤشش در تحقيق حاضر توسط كميته اخلاق در

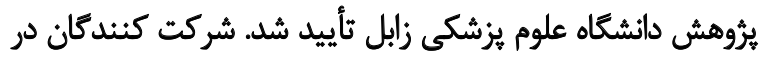

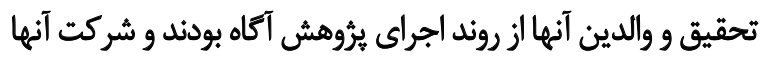

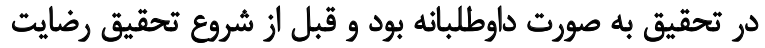

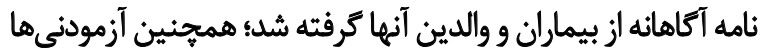

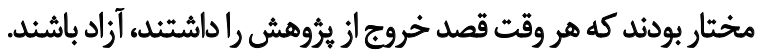

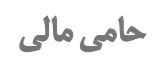

اين تحقيق هيجيَونه كمك مالى از سازمانهاى تأمين مالى در بخشهاى عمومى، تجارى يا غيرانتفاعى دريافت نكرد.

$$
\text { مشاركت نويسندكّان }
$$

مفهومسازي: اكبر قلاوند، حسين ساكى، فرزاد ناظم؛ تحقيق و

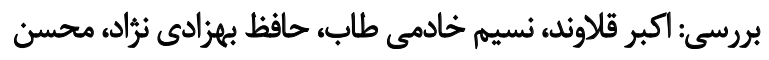
بجبودي، فاطمه ضيغمى؛ ويراستارى و نهايىسازى : اكبر قلاوند.

$$
\text { تعارض مثاقع }
$$

بنابر اظهار نويسندكان اين مقاله تعارض منافع ندارد. 


\section{Refrences}

[1] Tuomilehto J. The emerging global epidemic of type 1 diabetes. Curr Diab Rep. 2013; 13(6):795-804. [DOI:10.1007/ s11892-013-0433-5] [PMID]

[2] Derakhshan R, Bakhshi H. [The comparison study of magnesium serum between diabetic and non diabetic children (Persian)]. J Guilan Univ Med Sci. 2009; 18(71):90-4. http://journal.gums. ac.ir/article-1-243-en.html

[3] Hadiyan M. [Effectiveness of Acceptance \& Commitment Therapy (ACT) on separation anxiety disorders in children with type 1 diabetes (Persian)]. Razi J Med Sci. 2018; 24(164):21-34. http://rjms.iums.ac.ir/article-1-3928-en.html

[4] Cheraghi F, Mortazavi SZ, Shamsaei F, Moghimbeigi A. [Effect of education on management of blood glucose in children with diabetes (Persian)]. J Nurs Educ. 2014; 3(1):1-11. http:// jne.ir/article-1-286-en.html

[5] Carroll AE, Marrero DG. The role of significant others in adolescent diabetes: A qualitative study. Diabetes Educ. 2006; 32(2):243-52. [DOI:10.1177/0145721706286893] [PMID]

[6] Ríos JL, Francini F, Schinella GR. Natural products for the treatment of type 2 diabetes mellitus. Planta Med. 2015; 81(12/13):975-94. [DOI:10.1055/s-0035-1546131] [PMID]

[7] Ghalavand A, Delaramnasab M, Ghanaati S, Abdolahi gazari $M$. [Comparison of the effect of telenursing and aerobic training on cardiometabolic and anthropometric indices in patients with type 2 diabetes (Persiaan)]. Razi J Med Sci. 2021; 28(4):34-45. https://rjms.iums.ac.ir/article-1-6400-en.pdf

[8] Bernardini AL, Vanelli M, Chiari G, lovane B, Gelmetti C, Vitale $R$, et al. Adherence to physical activity in young people with type 1 diabetes. Acta Biomed. 2004; 75(3):153-7. [PMID]

[9] Riddell MC, Gallen IW, Smart CE, Taplin CE, Adolfsson P, Lumb $\mathrm{AN}$, et al. Exercise management in type 1 diabetes: A consensus statement. Lancet Diabetes Endocrinol. 2017; 5(5):377-90. [DOI:10.1016/S2213-8587(17)30014-1]

[10] Colberg SR, Laan R, Dassau E, Kerr D. Physical activity and type 1 diabetes: Time for a rewire? J Diabetes Sci Technol. 2015; 9(3):609-18. [DOI:10.1177/1932296814566231] [PMID] [PMCID]

[11] Wallberg-Henriksson H, Gunnarsson R, Rössner S, Wahren J. Long-term physical training in female type 1 (insulin-dependent) diabetic patients: Absence of significant effect on glycaemic control and lipoprotein levels. Diabetologia. 1986; 29(1):53-7. [DOI:10.1007/BF02427281] [PMID]

[12] Ramalho AC, de Lourdes Lima M, Nunes F, Cambuí Z, Barbosa C, Andrade A, et al. The effect of resistance versus aerobic training on metabolic control in patients with type- 1 diabetes mellitus. Diabetes Res Clin Pract. 2006; 72(3):271-6. [DOI:10.1016/j.diabres.2005.11.011] [PMID]

[13] Durak EP, Jovanovic-Peterson L, Peterson CM. Randomized crossover study of effect of resistance training on glycemic control, muscular strength, and cholesterol in type I diabetic men. Diabetes Care. 1990; 13(10):1039-43. [DOI:10.2337/diacare.13.10.1039] [PMID]
[14] Keypour S, Riahi H, Rafati H. [A review on the biological active compounds and medicinal properties of Ganoderma lucidum (Persian)]. J Med Plants. 2013; 12(46):13-24. http://jmp.ir/ article-1-85-en.html

[15] Ma HT, Hsieh JF, Chen ST. Anti-diabetic effects of Ganoderma lucidum. Phytochemistry. 2015; 114:109-13. [DOI:10.1016/j. phytochem.2015.02.017] [PMID]

[16] Meneses ME, Martínez-Carrera D, Torres N, Sánchez-Tapia $M$, Aguilar-López $M$, Morales $P$, et al. Hypocholesterolemic properties and prebiotic effects of Mexican Ganoderma lucidum in C57BL/6 Mice. PLoS One. 2016; 11(7):e0159631. [DOI:10.1371/journal.pone.0159631] [PMID] [PMCID]

[17] Xiao Ch, Wu Q, Zhang J, Xie Y, Cai W, Tan J. Antidiabetic activity of Ganoderma lucidum polysaccharides F31 downregulated hepatic glucose regulatory enzymes in diabetic mice. J Ethnopharmacol. 2017; 196:47-57. [DOI:10.1016/j. jep.2016.11.044] [PMID]

[18] Yardley JE, Kenny GP, Perkins BA, Riddell MC, Malcolm J, Boulay $P$, et al. Effects of performing resistance exercise before versus after aerobic exercise on glycemia in type 1 diabetes. Diabetes Care. 2012; 35(4):669-75. [DOI:10.2337/dc11-1844] [PMID] [PMCID]

[19] Ghalavand A, Delaramnasab M, Afshounpour M, Zare A. [Effects of continuous aerobic exercise and circuit resistance training on fasting blood glucose control and plasma lipid profile in male patients with type II diabetes mellitus (Persian)]. J Diabetes Nurs. 2016;4(1):8-19. http://jdn.zbmu.ac.ir/article1-146-en.html

[20] Salem MA, AboEIAsrar MA, Elbarbary NS, ElHilaly RA, Refaat YM. Is exercise a therapeutic tool for improvement of cardiovascular risk factors in adolescents with type 1 diabetes mellitus? A randomised controlled trial. Diabetol Metab Syndr. 2010; 2:47. [DOI:10.1186/1758-5996-2-47] [PMID] [PMCID]

[21] Qadir KJ, Zangana KO. Effect of swimming program on glycemic control in male adolescents with type 1 diabetes mellitus. The Journal of sports medicine and physical fitness. J Sports Med Phys Fitness. 2020; 60(2):302-7. [DOI:10.23736/S00224707.19.10053-9] [PMID]

[22] Tunar M, Ozen S, Goksen D, Asar G, Bediz CS, Darcan S. The effects of Pilates on metabolic control and physical performance in adolescents with type 1 diabetes mellitus. J Diabetes Complications. 2012; 26(4):348-51. [DOI:10.1016/j.jdiacomp.2012.04.006] [PMID]

[23] Fuchsjäger-Mayrl G, Pleiner J, Wiesinger GF, Sieder AE, Quittan M, Nuhr MJ, et al. Exercise training improves vascular endothelial function in patients with type 1 diabetes. Diabetes Care. 2002; 25(10):1795-801. [DOI:10.2337/diacare.25.10.1795] [PMID]

[24] Ghalavand A, Motamedi P, Delaramnasab M, Khodadoust $M$. The effect of interval training and nettle supplement on glycemic control and blood pressure in men with type 2 diabetes. Int J Basic Sci Med. 2017; 2(1):33-40. [DOI:10.15171/ ijbsm.2017.08] 
[25] Forga L, Goñi MJ, Chinchurreta L, Lafita FJ, Iriarte A, Fernández- Real JM. Acute exercise leads to increased HbA1c and fructosamine levels in athletes with type 1 diabetes. Endocr Abstr. 2013; 32:357. [DOI:10.1530/endoabs.32.P357]

[26] Admon G, Weinstein $Y$, Falk B, Weintrob N, Benzaquen $\mathrm{H}$, Ofan $\mathrm{R}$, et al. Exercise with and without an insulin pump among children and adolescents with type 1 diabetes mellitus. Pediatrics. 2005; 116(3):e348-55. [DOI:10.1542/peds.20042428] [PMID]

[27] Burns RD, Fu Y, Zhang P. Resistance training and insulin sensitivity in youth: A meta-analysis. Am J Health Behav. 2019; 43(2):228-42. [DOI:10.5993/AJHB.43.2.1] [PMID]

[28] Chaweewannakorn Ch, Nyasha MR, Chen W, Sekiai Sh, Tsuchiya M, Hagiwara Y, et al. Exercise-evoked intramuscular neutrophil-endothelial interactions support muscle performance and GLUT4 translocation: A mouse gnawing model study. J Physiol. 2020; 598(1):101-22. [DOI:10.1113/JP278564] [PMID]

[29] Horii N, Hasegawa N, Uchida M, lemitsu M. Increased muscle 5alpha-dihydrotestosterone by acute resistance exercise contributes to muscle GLUT4 signaling in diabetic rats. Med Sci Sports Exerc. 2019; 51(6S):498. [DOI:10.1249/01. mss.0000561995.99511.fe]

[30] Akbari N, Peeri M, Azarbayjani MA, Delfan M. [Comparison of the effect of 8 weeks of continuous and high intensity interval training on the gene expression of TIMP-2 and MMP-2 in male diabetic rats (Persian)]. Razi J Med Sci. 2019; 26(10):107-16. http://rjms.iums.ac.ir/article-1-5702-en.html

[31] Knudsen JR, Steenberg DE, Hingst JR, Hodgson LR, HenriquezOlguin $\mathrm{C}$, Li Zh, et al. Prior exercise in humans redistributes intramuscular GLUT4 and enhances insulin-stimulated sarcolemmal and endosomal GLUT4 translocation. Mol Metab. 2020; 39:100998. [DOI:10.1016/j.molmet.2020.100998] [PMID] [PMCID]

[32] Yardley JE, Iscoe KE, Sigal RJ, Kenny GP, Perkins BA, Riddell $M C$. Insulin pump therapy is associated with less post-exercise hyperglycemia than multiple daily injections: An observational study of physically active type 1 diabetes patients. Diabetes Technol Ther. 2013; 15(1):84-8. [DOI:10.1089/dia.2012.0168] [PMID]

[33] Steppel JH, Horton ES. Exercise in the management of type 1 diabetes mellitus. Rev Endocr Metab Disord. 2003; 4(4):355-60. [DOI:10.1023/A:1027302112655] [PMID]

[34] Firoozrai M, Nourbakhsh M, Razzaghi Azar M, Bastani AH. [Increase in erythrocyte membrane susceptibility and oxidative stress in patients with type I diabetes mellitus (Persian)]. Razi J Med Sci. 2004; 11(42):611-21. http://rjms.iums.ac.ir/article1-53-en.html

[35] Eroğlu HA, Beytut E. Effect of Ganoderma lucidum polysaccharides on oxidative damage in liver of STZ-diabetic rats. Biomed Res. 2018; 29(18):3436-43. [DOI:10.4066/biomedicalresearch.29-18-831]

[36] Li F, Zhang Y, Zhong Zh. Antihyperglycemic effect of Ganoderma lucidum polysaccharides on streptozotocin-induced diabetic mice. Int J Mol Sci. 2011; 12(9):6135-45. [DOI:10.3390/ ijms12096135] [PMID] [PMCID] 